Article

\title{
Fatty Acids Composition of Vegetable Oils and Its Contribution to Dietary Energy Intake and Dependence of Cardiovascular Mortality on Dietary Intake of Fatty Acids
}

\section{Jana Orsavova ${ }^{1}$, Ladislava Misurcova ${ }^{2, *}$, Jarmila Vavra Ambrozova ${ }^{2}$, Robert Vicha ${ }^{3}$ and Jiri Mlcek $^{2}$}

1 Language Centre, Faculty of Humanities, Tomas Bata University in Zlín, nám. T. G. Masaryka 5555, 76001 Zlín, Czech Republic; E-Mail: orsavova@fhs.utb.cz

2 Department of Food Analysis and Chemistry, Faculty of Technology, Tomas Bata University in Zlín, nám. T. G. Masaryka 5555, 76001 Zlín, Czech Republic; E-Mails: ambrozova@ft.utb.cz (J.V.A.); mlcek@ft.utb.cz (J.M.)

3 Department of Chemistry, Faculty of Technology, Tomas Bata University in Zlín, nám. T. G. Masaryka 5555, 76001 Zlín, Czech Republic; E-Mail: rvicha@ft.utb.cz

* Author to whom correspondence should be addressed; E-Mail: misurcova@ft.utb.cz; Tel.: +420-57-603-1592; Fax: +420-57-721-0172.

Academic Editor: Cenk Suphioglu

Received: 5 May 2015 / Accepted: 1 June 2015 / Published: 5 June 2015

\begin{abstract}
Characterizations of fatty acids composition in \% of total methylester of fatty acids (FAMEs) of fourteen vegetable oils - safflower, grape, silybum marianum, hemp, sunflower, wheat germ, pumpkin seed, sesame, rice bran, almond, rapeseed, peanut, olive, and coconut oil-were obtained by using gas chromatography (GC). Saturated (SFA), monounsaturated (MUFA) and polyunsaturated fatty acids (PUFA), palmitic acid (C16:0; 4.6\%-20.0\%), oleic acid (C18:1;6.2\%-71.1\%) and linoleic acid (C18:2; 1.6\%-79\%), respectively, were found predominant. The nutritional aspect of analyzed oils was evaluated by determination of the energy contribution of SFAs $(19.4 \%-695.7 \%$ ERDI), PUFAs (10.6\%-786.8\% ERDI), n-3 FAs (4.4\%-117.1\% ERDI) and n-6 FAs (1.8\%-959.2\% $\mathrm{E}_{\mathrm{RDI}}$ ), expressed in \% $\mathrm{E}_{\mathrm{RDI}}$ of $1 \mathrm{~g}$ oil to energy recommended dietary intakes (ERDI) for total fat (ERDI-37.7 kJ/g). The significant relationship between the reported data of total fat, SFAs, MUFAs and PUFAs intakes (\% ERDI) for adults and mortality caused by coronary heart diseases (CHD) and cardiovascular diseases (CVD) in twelve countries has not been confirmed by Spearman's correlations.
\end{abstract}


Keywords: vegetable oils; fatty acids; cardiovascular diseases; coronary heart diseases; Spearman's correlation

\section{Introduction}

Lipids are considered one of the most elemental nutrients for humans. Lipid metabolism generates many bioactive lipid molecules, which are fundamental mediators of multiple signaling pathways and they are also indispensable compounds of cell membranes. Any kind of changes in lipid metabolism can result in modification of membrane composition and subsequently in changes in its permeability. It may also lead to disruption of signaling networks and could be associated with some pathological states, such as cancer, cardiovascular, neurodegenerative, and metabolic diseases, and similarly with inflammatory complications [1-9]. Lipids consist of fatty acids (FAs) classified mostly according to the presence or absence of double bonds as saturated (SFAs - without double bonds), monounsaturated (MUFAs - with one double bond) and polyunsaturated fatty acids (PUFAs - with two or up to six double bonds); further, as cis or trans based on the configuration of the double bonds and as n-3 or n- 6 PUFAs depending on the position of the first double bond from the fatty acid methyl-end. The human body cannot synthesize PUFAs with the first double bond on C3 and C6 from the methyl-end because of the absence of appropriate enzymes. Thus, these fatty acids are essential (EFAs) and they have to be obtained from a diet, particularly by the consumption of fish and fish oils [1-5,10].

Unsaturated fatty acids can exist in a cis- or trans-configuration. The former configuration is found in most naturally occurring unsaturated fatty acids, the latter configuration is the result of technology processing, such as hydrogenation. Cis-unsaturated fatty acids are potent inducers of adiposomes known as lipid droplets, which have important roles in cell signaling, regulation of lipid metabolism and control of the synthesis and secretion of inflammatory mediators. Lipid droplets are sites for eicosanoid generation in cells during a process of inflammation and cancer [11].

Fundamental PUFAs are $\alpha$-linolenic (ALA, 18:3, n-3) and linoleic acid (LA, 18:2, n-6) from which other important PUFAs are derived [1]. Satisfactory transformation of ALA to docosahexaenoic acid (DHA, 22:6, n-3) depends on the activity of responsible $\Delta^{5}$ and $\Delta^{6}$ desaturases that could be influenced by several factors, such as dietary cholesterol and high-fat diet and also appears to be low in diabetics [12-14]. Further, decreasing effects on the $\Delta^{6}$ desaturase activity and therefore the conversions of LA and ALA to long chained polyunsaturated fatty acids (LCPUFAs) caused by low insulin levels, deficiency of protein and minerals, such as iron, zinc, copper, and magnesium have also been published [15]. Furthermore, conversion of dietary ALA into eicosapentaenoic acid (EPA, 20:5, n-3) is limited because of the competition for common desaturation and elongation enzymes of ALA and LA. Moreover, it has been proved that the affinity of $\Delta^{6}$ desaturase for ALA is greater than for LA [3].

Recently, essential fatty acids (EFAs) have been considered as functional food and nutraceuticals. A lot of research studies have documented their significant roles in many biochemical pathways resulting in cardioprotective effect because of their considerable antiatherogenic, antithrombotic, anti-inflammatory, antiarrhytmic, hypolipidemic effect, because of the potential of reducing the risk of serious diseases, especially cardiovascular diseases, cancer, osteoporosis, diabetes and other health 
promotion activities following from their complex influence on concentrations of lipoproteins, fluidity of biological membranes, function of membraned enzymes and receptors, modulation of eicosanoids production, blood pressure regulation, and finally, on the metabolism of minerals [1,16-21]. EPA and DHA have also been associated with the protection against mental disorders like Alzheimer's disease, aging and dementia, chronic daily headache and with attention-deficit hyperactivity disorder in children [22-24]. Biological activities of individual EFAs might be derived from the character and three-dimensional configuration of molecules and their subsequent enzymatic transformation in a wide scale of compounds named eicosanoids. Eicosanoids derived from n-6 and n-3 fatty acids have antagonistic effects. Eicosanoids from the first group promote an inflammation; the latter are much less inflammatory or even anti-inflammatory. Their concentration depends on the fatty acids amounts in diet and is also influenced by the competition between AA and EPA as substrates for specific enzymes - cyclooxygenases and 5-lipoxygenases [1,18,25].

Seriously, cardiovascular diseases have been documented to be the main cause of death in most Western countries. Coronary heart disease is closely connected with a progress of atherosclerosis evoked by the interactions between plasma lipids, lipoproteins, monocytes, platelets, endothelium, and smooth muscle of arterial walls resulting in narrowed coronary arteries [1]. Thus, dietary modulation with emphasis on the composition of dietary lipids could be a therapeutic option in the prevention of thrombosis and coronary infarctions and in the treatment of various diseases including hearth diseases to improve the quality of arterial walls and vascular patency. The important role of dietary pattern and lifestyle on human health has been often documented. 1.5 times higher blood level of trans fatty acids in younger Inuit compared to elder Inuit was observed in connection with a decrease in traditional food consumption accompanied with an increased consumption of supermarket food, i.e., Western diet [10]. Recently, nutritionists have recommended vegetable oils as an important part of a healthy diet due to their high contents of fatty acids (FAs) besides their traditional sources, such as fish oil and algae [1,26-28]. However, distribution and content of fatty acids differ in dependence on various plant sources of oils and technology process used for their production.

This paper evaluated FAs composition of some vegetable oils, energy contribution E (\% ERDI) of saturated (SFAs), polyunsaturated (PUFAs) fatty acids, n-3 PUFAs and n-6 PUFAs of analyzed oils to recommended dietary intakes for total fat (ERDI-37.7 kJ/g). The amounts (g) of SFAs, PUFAs, $\mathrm{n}-3$ PUFAs and n-6 PUFAs requisite to cover their recommended daily intakes were calculated using maximal values of recommended daily intakes for fatty acids (ERDI). Statistic evaluation of the relationship between reported data of total fat, SFAs, MUFAs and PUFAs intakes among adults in different countries and coronary heart diseases (CHD) and cardiovascular diseases (CVD) mortality by Spearman's correlations were computed.

\section{Results and Discussion}

\subsection{Fatty Acids Composition of Vegetable Oils}

Fourteen samples of analyzed vegetable oils were mostly produced as virgin oils with a sustentative content of monounsaturated (MUFAs) and polyunsaturated (PUFAs) fatty acids. They have been 
considered as functional food and used as an important part of a healthy diet. Their FAs compositions are presented in Table 1.

Table 1. Fatty acids composition of vegetable oils ${ }^{1}$.

\begin{tabular}{|c|c|c|c|c|c|c|c|c|c|c|c|c|c|c|}
\hline FAs [\%] & SAF & GRP & SIL & НМР & SFL & WHG & PMS & SES & RB & ALM & RPS & PNT & OL & $\mathrm{COC}$ \\
\hline C6:0 & nd & nd & nd & nd & nd & nd & nd & nd & nd & nd & nd & nd & nd & 0.52 \\
\hline C8:0 & nd & 0.01 & nd & nd & nd & nd & nd & nd & nd & nd & nd & nd & nd & 7.6 \\
\hline C10:0 & nd & nd & nd & nd & nd & nd & nd & nd & nd & nd & 0.01 & nd & nd & 5.5 \\
\hline C12:0 & nd & 0.01 & 0.01 & nd & 0.02 & 0.07 & nd & nd & nd & 0.09 & nd & nd & nd & 47.7 \\
\hline $\mathrm{C} 14: 0$ & 0.10 & 0.05 & 0.09 & 0.07 & 0.09 & nd & 0.17 & nd & 0.39 & 0.07 & nd & 0.04 & nd & 19.9 \\
\hline C15:0 & nd & 0.01 & 0.02 & nd & nd & 0.04 & nd & nd & nd & nd & 0.02 & nd & nd & nd \\
\hline C16:0 & 6.7 & 6.6 & 7.9 & 6.4 & 6.2 & 17.4 & 13.1 & 9.7 & 20.0 & 6.8 & 4.6 & 7.5 & 16.5 & nd \\
\hline C17:0 & 0.04 & 0.06 & 0.06 & 0.05 & 0.02 & 0.03 & 0.13 & nd & nd & 0.05 & 0.04 & 0.07 & nd & nd \\
\hline C18:0 & 2.4 & 3.5 & 4.5 & 2.6 & 2.8 & 0.7 & 5.7 & 6.5 & 2.1 & 2.3 & 1.7 & 2.1 & 2.3 & 2.7 \\
\hline C20:0 & nd & 0.16 & 2.6 & nd & 0.21 & nd & 0.47 & 0.63 & nd & 0.09 & nd & 1.01 & 0.43 & nd \\
\hline $\mathrm{C} 22: 0$ & nd & nd & nd & nd & nd & nd & nd & 0.14 & nd & nd & nd & nd & 0.15 & nd \\
\hline C16:1 (n-7) & 0.08 & 0.08 & 0.05 & 0.11 & 0.12 & 0.21 & 0.12 & 0.11 & 0.19 & 0.53 & 0.21 & 0.07 & 1.8 & nd \\
\hline C17:1 (n-7) & nd & nd & 0.03 & nd & nd & nd & nd & nd & nd & nd & nd & nd & nd & nd \\
\hline C18:1 cis (n-9) & 11.5 & 14.3 & 20.4 & 11.5 & 28.0 & 12.7 & 24.9 & 41.5 & 42.7 & 67.2 & 63.3 & 71.1 & 66.4 & 6.2 \\
\hline C18:1trans (n-9) & nd & nd & nd & nd & nd & nd & nd & nd & nd & nd & 0.14 & nd & nd & nd \\
\hline C20:1(n-9) & nd & 0.40 & 0.15 & 16.5 & 0.18 & 7.91 & 1.08 & 0.32 & 1.11 & 0.16 & 9.1 & nd & 0.30 & nd \\
\hline C18:2cis (n-6) & 79.0 & 74.7 & 63.3 & 59.4 & 62.2 & 59.7 & 54.2 & 40.9 & 33.1 & 22.8 & 19.6 & 18.2 & 16.4 & 1.6 \\
\hline C18:3 (n-3) & 0.15 & 0.15 & 0.88 & 0.36 & 0.16 & 1.2 & 0.12 & 0.21 & 0.45 & nd & 1.2 & nd & 1.6 & nd \\
\hline C18:3 (n-6) & nd & nd & nd & 3.0 & nd & nd & nd & nd & nd & nd & nd & nd & nd & nd \\
\hline SFAs & 9.3 & 10.4 & 15.1 & 9.2 & 9.4 & 18.2 & 19.6 & 16.9 & 22.5 & 9.3 & 6.3 & 10.7 & 19.4 & 92.1 \\
\hline MUFAs & 11.6 & 14.8 & 20.7 & 28.1 & 28.3 & 20.9 & 26.1 & 42.0 & 44.0 & 67.9 & 72.8 & 71.1 & 68.2 & 6.2 \\
\hline PUFAs & 79.1 & 74.9 & 64.2 & 62.8 & 62.4 & 61.0 & 54.3 & 41.2 & 33.6 & 22.8 & 20.9 & 18.2 & 18.0 & 1.6 \\
\hline n-3 PUFAs & 0.2 & 0.2 & 0.9 & 0.4 & 0.2 & 1.2 & 0.1 & 0.2 & 0.5 & 0.0 & 1.2 & 0.0 & 1.6 & 0.0 \\
\hline n-6 PUFAs & 79.0 & 74.7 & 63.3 & 62.4 & 62.2 & 59.7 & 54.2 & 40.9 & 33.1 & 22.8 & 19.6 & 18.2 & 16.4 & 1.6 \\
\hline
\end{tabular}

${ }^{1}$ Data are expressed as percentages of total fatty acid methyl esters (FAMEs); nd means that FAs was not determined. Abbreviations of the samples mean: SAF—safflower, GRP-grape, SIL—silybum marianum, HMP — hemp, SFL — sunflower, WHG — wheat germ, PMS — pumpkin seed, SES — sesame, RB — rice bran, ALM — almond, RPS — rapeseed, PNT — peanut, OL—olive, and COC—coconut oils.

Fatty acids composition of vegetable oils is formed by a mixture of saturated (SFAs) and unsaturated (UNFAs) fatty acids classified according to the number of unsaturated bonds as monounsaturated (MUFAs) or polyunsaturated fatty acids (PUFAs). Nevertheless, each of analyzed vegetable oils has specific fatty acid distribution depending on their plant sources. So, their impact on human health could be assessed according to individual fatty acids because of their different influences on human health and risks of serious diseases.

\subsubsection{Saturated Fatty Acids (SFAs)}

Saturated fatty acids with fewer than 12 carbon atoms being called short and medium chain saturated fatty acids (MCFAs) have been found only in coconut oil (COC) in the amount not exceeding $7.6 \%$ of total methylester of fatty acids (FAMEs) as can be seen in Table 1. Expectedly, SFAs were 
established as extraordinarily predominant FAs in the highest amount of $92.1 \%$ of total FAMEs in coconut oil (COC), and they were presented especially by lauric (C12:0) and myristic (C14:0) acids in the amounts of $47.7 \%$ and $19.9 \%$, respectively; in agreement with reported data [18,25,29-32]. In the rest of analyzed oils SFAs were determined in the range from $6.3 \%$ (rapeseed oil (RPS)) to 22.5\% (rice brain oil (RB)) of total FAMEs. Palmitic acid (C16:0) was found to be a predominant SFA in the majority of samples in the range from $4.6 \%$ (RPS) to $20.0 \%$ (RB). In short, observed contents of palmitic acid (C16:0) were also in agreement with reported data by [30-37].

In fact, some studies have reported various impacts of SFAs on the human health. It has been concluded that lauric acid (C12:0) as well as myristic acid (C14:0) raise plasma total cholesterol concentrations, the first due to an increase in LDL cholesterol, the latter due to a rise of both LDL and HDL cholesterol concentrations [38,39]. However, according to Mensink [40] and Lawrence [41], the ratio of total cholesterol to HDL cholesterol is a more specific marker of coronary artery diseases than the value of LDL cholesterol. Oils rich in lauric acid (C12:0) decreased the ratio of total to HDL cholesterol. On the other hand, myristic (C14:0) and palmitic acids (C16:0) affected this ratio only little and stearic acid (C18:0) slightly reduced this ratio.

\subsubsection{Monounsaturated Fatty Acids (MUFAs)}

The Mediterranean diet is well-known as a diet with high consumption of olive oil and minimal amount of saturated fatty acids. Red meat, whole fat milk products, nuts and high fat fruits, such as olives and avocados are among the natural sources of MUFAs. The majority of all investigated samples, except for coconut oil, showed the highest proportion of MUFAs or PUFAs in their FAMEs composition. In general, MUFAs were distributed mostly in higher amounts than SFAs in the range from $6.2 \%$ to $72.8 \%$ in coconut (COC) and rapeseed (RPS) oils, respectively. Interestingly, MUFAs formed a main part of fatty acid compositions in six analyzed oils, such as $72.8 \%$ in rapeseed oil (RPS), $71.1 \%$ in peanut oil (PNT), 68.2\% in olive oil (OL), $67.9 \%$ in almond oil (ALM), $44.0 \%$ in rice brain oil (RB), and $42.0 \%$ in sesame oil (SES). Oleic acid (C18:1, n-9) was found as the most abundant MUFA in almost all samples in the range from $6.2 \%$ (COC) to $71.1 \%$ (PNT), except for hemp oil (HMP), where eicosenoic acid (C20:1, n-9) was established as the predominant MUFA in the amount of $16.5 \%$ conversely to reported data by [30], where eicosenoic acid in hemp oil was not determined. Observed contents of oleic acid in the selected samples of vegetable oils were significantly in accordance with reported values [30-37] with the exception of oleic acid content in peanut oil (PNT) that was higher in comparison to reported data [30,32]. It has been documented that MUFAs may reduce LDL cholesterol, while it might possibly increase high-density lipoprotein (HDL) cholesterol [15]. Oleic acid (C18:1, n-9) may promote insulin resistance contrary to PUFAs with the protection against insulin resistance [15]. Further, the saturation index (SI) in red blood cell membranes is formed by the ratio of stearic (C18:0, SFA) to oleic acid (C18:1, n-9, MUFA) and it is found as an appropriate biomarker for investigating the relation between the pattern of metabolism and breast cancer risk [42]. Oleic acid has also been reported as anti-apoptotic and anti-inflammatory agent via down regulation of cyclooxygenase-2 (COX-2) and inducible nitric oxide synthase (iNOS) through the activation of nuclear factor-kappa $\mathrm{B}(\mathrm{NF}-\kappa \mathrm{B})$ resulting in the activation of downstream inflammatory mediators [43]. 


\subsubsection{Polyunsaturated Fatty Acids (PUFAs)}

Primary source of PUFAs, especially docosahexaenoic acid (DHA, C22:6, n-3) and linoleic acid (LA, C18:2, n-6) is algae and marine phytoplankton forming the main part of fish feed as was previously described by $[1,44-46]$. Terrestrial sources are found mostly in nuts, seeds, and leafy vegetables [47]. In seven analyzed oils_-safflower oil (SAF), grape oil (GRP), silybum marianum oil (SIL), hemp oil (HMP), sunflower oil (SFL), wheat germ (WGM), and pumpkin seed oil (PMS), PUFAs presented as the predominant part of fatty acid compositions and their contents ranged from $54.3 \%$ in pumpkin seed oil (PMS) to $79.1 \%$ in safflower oil (SAF). The most abundant PUFA was linoleic acid (LA, C18:2, n-6) in all analyzed samples, in the range from 1.6\% in coconut oil (COC) to $79.0 \%$ in safflower oil (SAF). Similar results of LA (C18:2, n-6) have been reported for grape, almond, wheat germ, sesame, pumpkin seed and safflower oil [33]; for peanut, rapeseed and coconut oil [30,32]; and finally for hemp oil [30], rice brain oil [31], sunflower oil [31,37] and olive oil [37,48]. However, the obtained content of $63.3 \%$ of LA $(\mathrm{C} 18: 2, \mathrm{n}-6)$ in SIL was higher than the published amount [35].

Recent studies have clearly shown the important impact of polyunsaturated fatty acids (PUFAs) on human health in the prevention of, particularly, cardiovascular disease (DVD), coronary heart disease and cancer; further, inflammatory, thrombotic and autoimmune disease; hypertension; diabetes type two, renal diseases; and rheumatoid arthritis, ulcerative colitis, and Crohn's disease. Their non-substitutable roles in many biological pathways are crucial $[49,50]$.

The difference between the locations of the first double bond in the fatty acid carbon chain (n-3 and $\mathrm{n}-6$ PUFAs) is the reason of significant differences in their biological functions that might be derived from the course of their interactions [1]. The $n-6 / n-3$ ratio is considered to be the key factor for balanced synthesis of eicosanoids and its nutritional importance has been frequently discussed as well as dependence of n-6/n-3 ratio value on a dietary pattern. High consumption of plant oils rich in n-6 PUFAs and relatively low consumption of marine fish products could cause this ratio to be too high, mainly seen in Western countries. Though, industrially produced food with high content of n-6 PUFAs also acquired popularity in the regions with traditional dietary patterns characterized by low blood value of n-6/n-3 ratio [1,10,49]. In the analyzed oils, n-3 PUFAs represented by $\alpha$-linolenic acid (ALA, C18:3, n-3) were found in the range of $0.1 \%-1.6 \%$, except for PNT, ALM and COC oils, where n-3 PUFAs were not determined. The group of n-6 PUFAs was represented by linoleic (LA, C18:2, n-6) and $\gamma$-linolenic (GLA, C18:3, n-6) acids. Whereas LA was found to be the predominant PUFAs in the analyzed samples in the range of $1.6 \%-79.0 \%$, GLA was found only in sHMP in the amount of $3.0 \%$.

\subsection{Contribution of Fatty Acid Groups of Vegetable Oils to Energy Daily Intake}

Lipids belong to main sources of energy for human metabolic processes. Lipid consumption in most Western countries is relatively high with the contribution of approximately $40 \%$ of total calories [51]. This is despite the nutritional recommendation that $25 \%$ of energy should be covered by lipids [52] and total fat daily intake of $20 \%-35 \%$ of energy is recommended by Food and Agriculture Organization (FAO) of the United Nations and the World Health Organization (WHO) [15]. Nevertheless, variations in the structures of individual fatty acids appear to elicit their different physiological functions with diverse impacts on human health. However, the absolute dietary intake of 
lipids is not considered as the main promoter of cardiovascular diseases. Relative concentration and distribution of fatty acids in dietary fats have been reported to be an important factor in considering nutritional values of lipids as well as the key factor, with proved effects, of lowering the risk of cardiovascular diseases [1,53]. In Table 2 the energy values $(\mathrm{kJ} / \mathrm{g})$ calculated from SFAs, MUFAs, PUFAs, n-3 PUFAs and n-6 PUFAs contents of fourteen vegetable oils using the conversion factor of $37.7 \mathrm{~kJ} / \mathrm{g}$ for fat and fatty acids are shown.

Table 2. Energy values $(\mathrm{E}, \mathrm{kJ} / \mathrm{g})$ of different fatty acid groups derived from $1 \mathrm{~g}$ of vegetable oils ${ }^{1}$.

\begin{tabular}{|c|c|c|c|c|c|c|c|c|c|c|c|c|c|c|}
\hline$E(k J / g)$ & SAF & GRP & SIL & HMP & SFL & WHG & PMS & SES & RB & ALM & RPS & PNT & OL & $\mathrm{COC}$ \\
\hline SFAs & 2.9 & 2.6 & 5.0 & 3.0 & 4.7 & 4.1 & 4.6 & 3.5 & 6.2 & 2.1 & 4.4 & 3.2 & 0.7 & 26.2 \\
\hline MUFAs & 3.7 & 3.8 & 6.0 & 9.5 & 14.6 & 4.8 & 8.9 & 8.1 & 12.3 & 16.4 & 47.4 & 22.0 & 2.6 & 1.7 \\
\hline PUFAs & 26.0 & 19.7 & 19.0 & 21.1 & 32.6 & 14.2 & 12.5 & 9.4 & 9.4 & 5.5 & 13.6 & 5.7 & 0.7 & 0.4 \\
\hline n-3 PUFAs & 0.1 & 0.0 & 0.4 & 0.1 & 0.1 & 0.3 & 0.0 & 0.0 & 0.1 & 0.0 & 0.9 & 0.0 & 0.0 & 0.0 \\
\hline n-6 PUFAs & 25.9 & 19.6 & 18.6 & 20.9 & 32.5 & 13.9 & 12.4 & 9.4 & 9.3 & 5.5 & 12.8 & 5.7 & 0.1 & 0.4 \\
\hline
\end{tabular}

${ }^{1}$ Data are expressed as energy values (E) in $\mathrm{kJ} / \mathrm{g}$ of different fatty acid groups of SFAs, MUFAs, PUFAs, $\mathrm{n}-3$ and n-6 PUFAs derived from $1 \mathrm{~g}$ of analyzed oils.

Moreover, for an easy evaluation of nutritional aspect of analyzed vegetable oils from the energy contribution E (\% ERDI) of their SFAs, PUFAs, n-3 PUFAs and n-6 PUFAs point of view, the energy values $(\mathrm{kJ} / \mathrm{g}$ oil) from Table 2 were converted into the percentages of recommended daily intakes for total fat (ERDI-37.7 kJ/g) for different fatty acid groups by using the maximal values of recommended daily intakes of ERDI-SFAs - 10\% ERDI; ERDI-PUFAs - 11\% ERDI; ERDI-n-3-2\% ERDI and ERDI-n-6-9\% ERDI according to FAO/WHO [15]. Data are shown in Table 3.

Table 3. Percentage contribution of energy (E) of SFAs, PUFAs, n-3 PUFAs and n-6 PUFAs of vegetable oils to recommended daily intakes for total fat $\left(\mathrm{E}_{\mathrm{RDI}}-37.7 \mathrm{~kJ} / \mathrm{g}\right)^{1}$.

\begin{tabular}{|c|c|c|c|c|c|c|c|c|c|c|c|c|c|c|}
\hline E [\% ERDI] & SAF & GRP & SIL & НМР & SFL & WHG & PMS & SES & RB & ALM & RPS & PNT & OL & $\mathrm{COC}$ \\
\hline SFAs & 77.2 & 69.9 & 132.2 & 78.5 & 124.9 & 107.5 & 121.3 & 93.9 & 163.4 & 56.7 & 116.2 & 84.2 & 19.4 & 695.7 \\
\hline PUFAs & 626.3 & 474.5 & 457.1 & 507.9 & 786.8 & 342.3 & 300.9 & 226.9 & 227.2 & 132.7 & 329.1 & 137.4 & 16.4 & 10.6 \\
\hline n-3 PUFAs & 6.7 & 5.5 & 47.8 & 17.0 & 11.2 & 41.1 & 5.0 & 4.4 & 17.6 & 0.0 & 117.1 & 0.0 & 0.0 & 0.0 \\
\hline n-6 PUFAs & 764.0 & 578.7 & 547.6 & 617.0 & 959.2 & 409.3 & 366.6 & 276.4 & 273.8 & 162.2 & 376.2 & 167.9 & 1.8 & 13.0 \\
\hline
\end{tabular}

${ }^{1}$ Data are expressed as percentages of recommended daily intakes for total fat $\left(\mathrm{E}_{\mathrm{RDI}}-37.7 \mathrm{~kJ} / \mathrm{g}\right) \mathrm{calculated}$ by using maximal values of recommended daily intakes of $\mathrm{E}_{\mathrm{RDI}-\mathrm{SFAs}}-10 \% \mathrm{E}_{\mathrm{RDI}}$; $\mathrm{E}_{\mathrm{RDI}-\mathrm{PUFAs}}-11 \% \mathrm{E}_{\mathrm{RDI}}$; $\mathrm{E}_{\mathrm{RDI}-\mathrm{n}-3}$ PUFAs $-2 \% \mathrm{E}_{\mathrm{RDI}}$ and $\mathrm{E}_{\mathrm{RDI}-\mathrm{n}-6 \mathrm{PUFAs}}-9 \% \mathrm{E}_{\mathrm{RDI}}$.

According to the recommendations of the Report of an expert consultation of FAO/WHO [15] for adults, total intake of SFAs should not exceed 10\% ERDI. Recommended MUFAs daily intake has not been given by definite number, but it is calculated from the difference between total fat intake ( $\%$ ERDI) and the amounts of SFAs, PUFAs, and trans-fatty acids (TFAs) in \% ERDI. Further, recommended daily energy intake of total PUFAs is in the range of 6\%-11\% ERDI. Total n-3 PUFAs energy intake should be in the range of $0.5 \%-2 \%$ ERDI. Total n-6 PUFAs energy intake is $2.5 \%-9 \%$ ERDI, and finally for TFAs, daily energy intake is lesser than $1 \%$ ERDI [15].

From results given in Table 3 could be concluded that energy equivalents corresponding SFAs, PUFAs, n-3 PUFAs and n-6 PUFAs energy intakes derived from $1 \mathrm{~g}$ of almost all analyzed oils exceeded 
general recommendations of the Report of an expert consultation of FAO/WHO for adults [15]. Energy values of SFAs ranged from 19.4\% ERDI (OL) to 695.7\% ERDI (COC). Warningly, the highest value of energy derived from SAFs in $1 \mathrm{~g}$ of COC exceeded the recommended maximum of $10 \%$ ERDI by almost seventy times. Thus, despite the production of new food products containing coconut oil, its extensive consumption has not been recommended from the view of the hypercholesterolemic effect.

Due to the fact that recommended MUFAs intake has not been given by definite number, energy originating from MUFAs is not included in Table 3.

Similar results of energy values of PUFAs were obtained; they varied between $10.6 \%$ ERDI (COC) and $786.8 \%$ ERDI (SFL). Importantly, analyzed oil samples represent an abundant source of PUFAs as it is evident from obtained results. Energy contribution of PUFAs derived from $1 \mathrm{~g}$ oils in the majority of samples multiply exceeded recommended value of $11 \%$ ERDI except for OL with a slightly higher amount of $16.4 \%$ ERDI and also COC with a value of 10.6\% ERDI. Enormous providers of energy derived from PUFAs, besides SFL mentioned above, are also these oils: SAF (626.3\% ERDI), HMP (507.9\% ERDI), GRP (474.5\% ERDI), SIL (457.1\% ERDI), WHG (342.3\% ERDI), RPS (329.1\% ERDI), PMS (300.9\% $\left.\mathrm{E}_{\mathrm{RDI}}\right), \mathrm{RB}(227.2 \% \mathrm{ERDI})$ and SES (226.9\% ERDI). Energy values of PNT and ALM performed 137.4\% ERDI and 132.7\% ERDI, respectively. Since the energy intake from PUFAs higher than 11\% ERDI could be a potential risk of lipid peroxidation, the consumption of these oils should be rather in minor amounts and less frequent.

Energy values derived from the last two groups of n-3 PUFAs and n-6 PUFAs also exceeded general recommendations of their energy intakes derived from $1 \mathrm{~g}$ oils except for ALM, PNT, OL and COC, where n-3 PUFAs were not determined. Further, if compared to FAO/WHO recommendation for ALA intake of $0.5 \%-2 \%$ ERDI for adults, established amounts of $\alpha$-linolenic acid (ALA, C18:3, n-3) as the main representative of n-3 PUFAs in the rest of oils showed higher energy contribution in the range

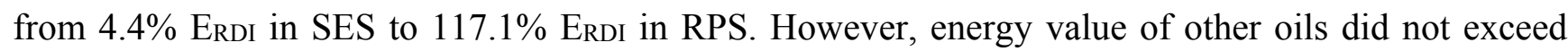
$50 \%$ ERDI- $47.8 \%$ ERDI in SIL and $41.1 \%$ ERDI in WHG.

Contrary to n-3 PUFAs, large contribution of n-6 PUFAs to energy intake has been revealed in the range from $1.8 \% \mathrm{E}_{\mathrm{RDI}}(\mathrm{OL})$ to $959.2 \% \mathrm{E}_{\mathrm{RDI}}$ (SFL). According to FAO/WHO, recommended intake of significant n-6 PUFA-LA for adults is 2.5\%-9\% ERDI. With the only exception of OL, in all other analyzed oils LA multiple exceeded this recommended amount, e.g., 764.0\% ERDI (SAF), 617.0\% ERDI (HMP), 578.7\% ERDI (GRP), 547.6\% ERDI (SIL), and 409.3\% ERDI (WHG), which is in contrast with insignificant contribution to energy intake of n-6 PUFAs of OL and 13\% ERDI (COC) mentioned above.

Trans elaidic acid (C18:1, n-9) is the principal trans-unsaturated fatty acid often found in partially hydrogenated vegetable oils [54]. It was determined only in RPS and its energy contribution represented a higher amount of $2.7 \%$ ERDI in relation with recommended daily intake of less than $1 \%$ ERDI for TFAs (result was not shown in Table 3).

\subsection{Oil Amounts Covering Recommended Daily Intakes of SFAs, PUFAs, n-3 and n-6 PUFAs}

It is evident that the amounts of analyzed oils for fulfillment of recommended energy daily intakes of individual FAs groups vary according to their specific FAs composition depending on specific plant source. In Figures 1 and 2 are shown the amounts $(\mathrm{g})$ of analyzed vegetable oils that is needed to cover 
recommended energy daily intakes of SFAs and PUFAs (Figure 1) and further recommended energy daily intakes of n-3 PUFAs and n-6 PUFAs (Figure 2).

Oil amounts covering recommended energy daily intake (g) of SFAs and PUFAs

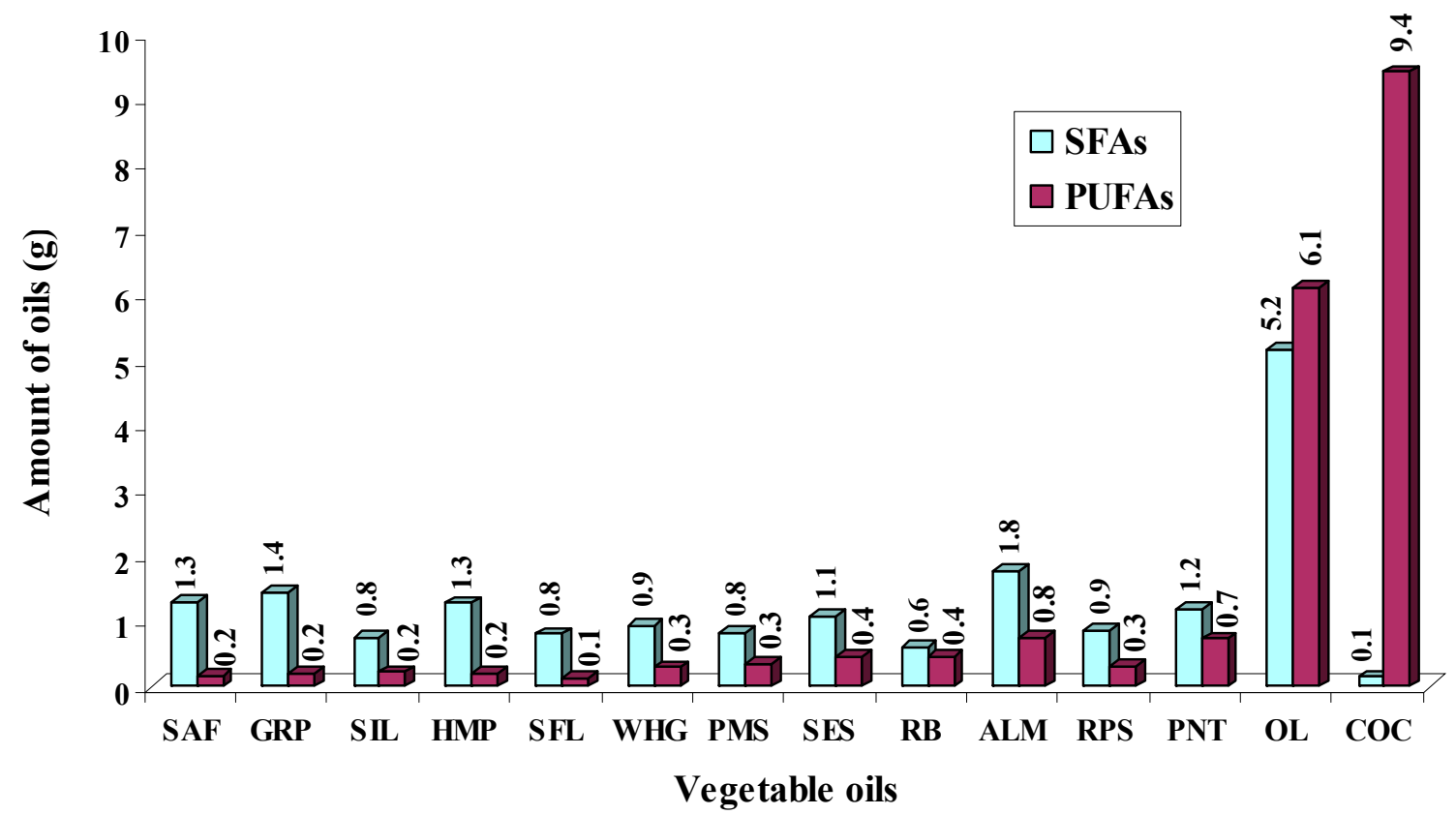

Figure 1. Oil amounts (g) needed to cover recommended energy daily intakes of SFAs and PUFAs calculated from their maximal recommended daily values (ERDI).

Oil amounts covering recommended energy daily intake (g) of n-3 and n-6 PUFAs

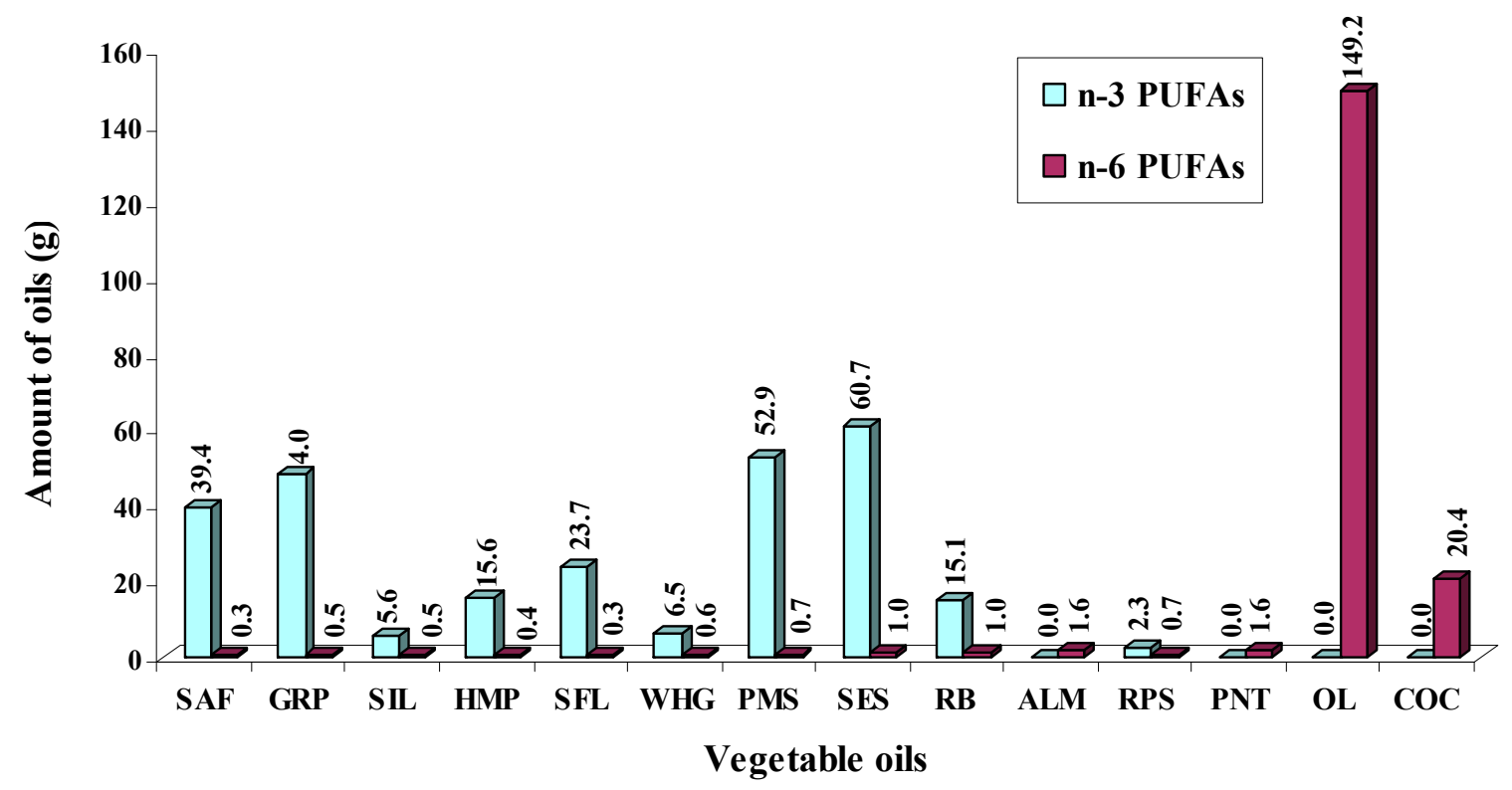

Figure 2. Oil amounts (g) needed to cover recommended energy daily intakes of n-3 PUFAs and n-6 PUFAs calculated from their maximal recommended daily values (ERDI). 
For coverage of SFAs energy recommended daily intake are needed oil amounts in the range from $0.1 \mathrm{~g}(\mathrm{COC})$ to $5.2 \mathrm{~g}(\mathrm{OL})$. Since the analyzed oils were mostly established as important sources of PUFAs, the amounts needed to cover their recommended energy daily intake are relatively low and in the majority of oils it ranged between $0.1 \mathrm{~g}$ (SFL) and $0.8 \mathrm{~g}$ (ALM) with the exception of the amount of $9.4 \mathrm{~g}$ and $6.1 \mathrm{~g}$ in COC and OL that are both an abundant source of SFAs and MUFAs.

Importantly, analyzed oils were abundant in contents of n-6 PUFAs, so the amounts needed to cover their recommended daily intakes would have to be small and ranged from $0.3 \mathrm{~g}$ (SFL and SAF) to $1.6 \mathrm{~g}$ (PNT and ALM). With the exception of OL rich in oleic acid (MUFAs) whose amount of $20.4 \mathrm{~g}$ is needed to cover recommended energy daily intake of n-6 PUFAs and COC, which is an abundant source of SFAs, but deficient in n-6 PUFAs, so the amount to cover recommended energy daily intake for n-6 PUFAs is $149.2 \mathrm{~g}$.

Finally, analyzed oils were characterized by mostly a small content of n-3 PUFAs, thus the amounts needed to cover the recommended daily intakes were relatively large from $2.3 \mathrm{~g}$ (RPS) to $60.7 \mathrm{~g}$ (SES).

\subsection{Statistical Evaluation of Impact of Fat Dietary Intake on the Number of CHD and CVD Mortality in Various Countries}

The role of dietary fats in cardiovascular disease (CVD) and many other disorders has been often discussed with general conclusions that this process is not simple, but rather a consequence of complex factors. Nevertheless, each group of fatty acids_-SFAs, MUFAs, PUFAs and individual FAs — has a specific role in many biopathways and imbalance in their dietary intake could be result in many serious diseases. Coronary heart diseases (CHD) induce the biggest mortality worldwide. The major risk factors are contemporary lifestyle characteristic in physical training deficit, smoking, excessive food intake and wrong dietary pattern leading to overweight or obesity, high blood pressure, high level of total cholesterol, LDL cholesterol, and triglycerides [55]. In this section, statistical evaluation of impact of fat dietary intakes on CHD and CVD mortality in various countries is provided by using coronary heart disease (CHD) number and cardiovascular disease (CVD) mortality per 100,000 inhabitants and intakes of total fats, SFAs, MUFAs, and PUFAs of an adult in different countries. Data about CHD and CVD mortality were collected from the sources [56-60] and intakes of total fats, SFAs, MUFAs and PUFAs in different countries were used according to [61], all shown in Table 4.

As seen in Table 4, it could be concluded different intakes (\% ERDI) of total fats, SFAs, MUFAs and PUFAs in some European countries (the Czech Republic, Germany, Austria, Finland, United Kingdom, Norway, France and Greece), the USA, the Republic of South Africa, Australia with New Zealand, and Japan. Generally, a lower intake of SFAs was mostly in relation with a lower intake of total fat. The highest intakes of SFAs in some European countries and the USA could give evidence to popularity of hydrogenated fats intakes; similarly, the highest MUFAs intake in Greece is probably in connection with the Mediterranean dietary pattern characterized by high consumption of olive oil. Documented intakes of PUFAs are very heterogeneous in the range of 3.9\%-8.0\% ERDI and are not in relation with number of CHD and CVD mortality in monitored countries. In accordance with data from Table 4, relatively high intakes of total fats were documented in global perspective study by Elmadfa and Kornsteiner [55] in Europe in the range of 28.5\%-46.2\% ERDI, but also in Africa 13.1\%-50.7\% ERDI, and America 25.7\%-37.2\% ERDI. In the other continents-Asia and Australia-they were in the 
ranges of $11.1 \%-35.6 \%$ ERDI and $32.5 \%-35.0 \%$ ERDI, respectively. According to [55], the highest intakes of MUFAs 10.9\%-22.3\% ERDI were also reported in Europe, further in Australia in the range of $11.8 \%-12.0 \%$ ERDI, while the highest PUFAs intakes were $3.3 \%-11.3 \%$ ERDI in Asia, which could be a consequence of typical cuisine patterns with high consumption of fish oil with the high content of PUFAs.

Table 4. CHD and CVD mortality and intakes of total fats, SFAs, MUFAs and PUFAs of an adult in various countries.

\begin{tabular}{cccccc}
\hline \multirow{2}{*}{ Country } & $\begin{array}{c}\text { CHD + Other CVD } \\
\text { Mortality Per 100,000 }\end{array}$ & Total Fats & SFAs & MUFAs & PUFAs \\
\cline { 3 - 6 } & 388.27 & 36.0 & 13.0 & 13.0 & 7.0 \\
Czech Republic & 364.75 & 35.9 & 14.4 & 12.8 & 6.5 \\
Germany & 320.92 & 37.0 & 14.5 & 12.5 & 8.0 \\
Austria & 284.69 & 32.1 & 13.5 & 12.4 & 6.2 \\
Finland & 260.58 & 34.0 & 11.0 & 12.5 & 7.0 \\
USA & 243.45 & 32.9 & 12.0 & 11.7 & 5.9 \\
United Kingdom & 242.72 & 30.6 & 12.1 & 10.8 & 5.4 \\
Norway & 237.45 & 27.7 & 8.6 & 9.5 & 6.9 \\
Republic of South Africa & 181.40 & 37.2 & 14.1 & 11.8 & 3.9 \\
France & 162.44 & 33.1 & 12.9 & 12.1 & 5.0 \\
Australia, New Zealand & 141.04 & 46.2 & 13.1 & 22.3 & 6.6 \\
Greece & 28.00 & 25.3 & 8.4 & 9.4 & 7.5 \\
Japan & &
\end{tabular}

${ }^{1}$ Data were collected from the sources [56-60]; ${ }^{2}$ Data of total fats, SFAs, MUFAs and PUFAs intakes in different countries were used according to [61].

Relatively high number of CHD and CVD mortality has been found in some European countries, especially in the Czech Republic, Germany and Austria, with values exceeding three hundred events per 100,000 habitants. However, not only total fat intakes, but other factors, such as dietary pattern and stressful life style, and amounts and distribution of fatty acids could contribute to CHD and CVD mortality. The impossibility of recognizing direct relationships between total fats, SFAs, MUFAs and PUFAs intakes and CHD and CVD mortalities in different regions based on data from Table 4 is evident. Thus, in spite of the highest consumption of total fat in Greece, only a relatively low value of CHD and CVD mortality was documented. Similarly, the highest PUFAs intake in Austria did not correlate with the high number of CHD and CVD mortality.

In Figure 3, dependence of CHD and CVD mortality on total fats, SFAs, MUFAs and PUFAs intakes is demonstrated. In the case of MUFAs, two correlations were performed; in MUFAs 1 (correlation C) all monitored countries were included, while for MUFAs 2 (correlation E) it was created without data regarding Greece with a specific high consumption of olive oil, an abundant source of MUFAs. Statistical analyses using Spearman's correlation have not confirmed the significant relationship between CHD and CVD mortalities and intakes (\% ERDI) of total fats, SFAs, MUFAs 1 and PUFAs in various countries. However, providing data from Greece were excluded, correlation E between MUFAs 2 intake and mortality showed even higher significance than correlation between SFAs and mortality. It is evident that direct dependence between factors mentioned above does not exist. The impact of specific fatty acids on disease incidence is very difficult to explain because of 
numerous factors including lifestyle as well as various functions and relationships between individual fatty acids in human biochemical pathways.
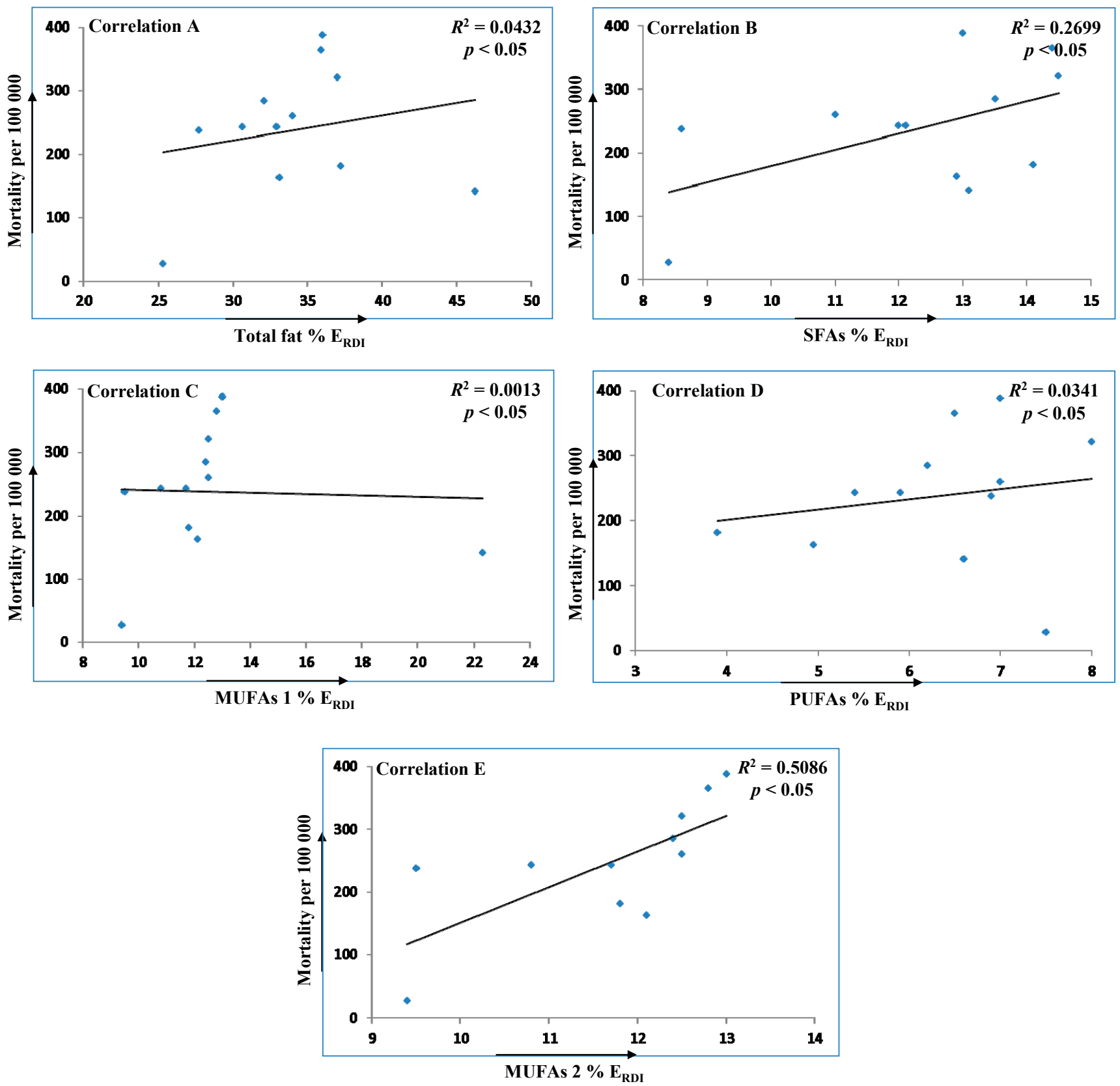

Figure 3. Spearman's correlations illustrating relationships between CHD and CVD mortality per 100,000 people and intakes (\% ERDI) of total fat (A); SFAs (B); MUFAs 1 (C); PUFAs (D) and MUFAs 2 (E) in various countries. MUFAs 1 is correlation including all countries, whereas MUFAs 2 is created without data from Greece.

Results of prospective epidemiologic studies have proved the correlation between specific types of fats and risk of CHD, but no relationships with total fats [20,62]. High level of CHD and CVD mortality in Western countries has provoked general nutritional recommendations to reduce dietary intake of SFAs as cardinal prevention of these diseases. However, conclusions from other studies have indicated that dietary SFAs reduction itself cannot suppress the risk of CHD and CVD incidence and 
mortality and do not clearly support cardiovascular guidelines promoting high consumption of n-6 PUFAs and suggest reduced consumption of SFAs [63,64]. A complex solution is needed. Mostly, reduction of SFAs and TFAs with their simultaneous replacement by PUFAs could lead to reduction of the risk of CHD [61]. However, some argue that impact of excessive consumption of n-6 PUFAs on risks of CVD leads to fixing an upper limit of ideally about 10\% E [65]. In another systematic analysis focused on global, regional and national consumption levels of dietary fats and oils in 1990 and 2010, great differences in fatty acids dietary intakes were also stated [66].

Moreover, reduction of one macronutrient in the diet has to be accompanied by an increase of another macronutrient to maintain the energy balance. Mostly, replenishment of energy after a decrease of fat intakes is accompanied with an increase of carbohydrate intakes. However, higher sugar levels in food do not prevent the increasing trend towards obesity and the second type of diabetes. Short-chain SFAs, e.g., from dairy fat and coconut oil, can influence gene expression via interactions with various G-protein-coupled receptors responsible for several hormonal responses, including insulin and leptin regulating overall energy metabolism in the human body [41]. Low-fat, high-carbohydrate diets are known to reduce HDL cholesterol and to raise triglycerides associated with increased CHD risk [40,55]. Further, cohort studies have showed an influence of different dietary composition on CHD risk. Replacing SFAs with PUFAs was inversely associated with the risk of CHD, whereas replacing SFAs with carbohydrates was not associated with CHD risk [67].

What is more, the study of almost 80 thousand women performed age-analyses and body mass index-analyses and proved the inverse correlation between polyunsaturated fat intake and CHD risk, while the trans-fat intake was associated with the raised risk of CHD. The strongest association was among women younger than 65 and also in women with the body mass index higher than $25 \mathrm{~kg} / \mathrm{m}^{2}$ [62]. Obesity and insulin resistance are both powerful predictors of CHD risk. Possibly, PUFAs can suppress the risk of CHD by improving both blood lipid levels and insulin resistance in overweight women, whilst TFAs may influence risk of CHD by its adverse effects on blood lipid levels [62,68].

Findings from many cohort studies are apparently inconsistent. Importantly, studies focused on the relationship between macronutrient intake and CHD have to be multifactorial as many environmental factors and personal conditions influence individual metabolic pathways. That is why the simple relationship between, i.e., total fats, SFAs and PUFAs intakes and the risk of serious diseases could not be determined.

\section{Experimental Section}

\subsection{Samples}

The study was conducted on fourteen vegetable oils purchased in a specialized local store (Table 5). 
Table 5. Description of analyzed samples of vegetable oils.

\begin{tabular}{cccc}
\hline Oils & Samples & Plant Sources & Technological Process \\
\hline Grape & GRP & Vitis vinifera & - \\
Peanut & PNT & Arachis hypogaea & - \\
Rapeseed & RPS & Brassica napus & Cold drawn \\
Sunflower & SFL & Helianthus annuus & Cold drawn \\
Safflower & SAF & Carthamus tinctorius & Cold drawn \\
Almond & ALM & Amygdalus communis & Cold drawn \\
Wheat germ & WHG & Triticum aestivum & Cold drawn \\
Sesame & SES & Sesamum indicum & Cold drawn \\
Pumpkin seed & PMS & Cucurbita pepo & Cold drawn \\
Rice brain & RB & Oryza sativa & - \\
Silybum marianum & SIL & Silybum marianum & Cold drawn \\
Coconut & COC & Cocos nucifera & - \\
Hemp & HMP & Cannabis sativa & Cold drawn \\
Olive & OL & Olea europaea & Cold drawn \\
\hline
\end{tabular}

\subsection{Standards and Reagents}

The standard mixture of 37 FAMEs (FAME Mix, SUPELCO, Bellefonte, PA, USA), and methyl-undecanoate (which was used as the internal standard) were purchased from Sigma Aldrich Chemical Co. (St. Louis, MO, USA). All other used chemicals were purchased from Merck (Darmstadt, Germany) and were of analytical purity.

\subsection{Preparation of FAMEs}

Firstly, fatty acids of oils have to be converted in their methyl esters (FAMEs) before gas chromatography (GC) analyses with a modification according to valid standard method in the Czech Republic [69] are performed. This method with usage of acid catalyst boron trifluoride is supported by the fact that basic catalysis does not convert free fatty acids especially in oils as can be concluded from the paper [70]. Briefly, $4 \mathrm{~mL}$ of $0.5 \mathrm{M}$ sodium hydroxide in methanol were added to $1 \mathrm{~mL}$ oil sample, the equipment was closed and heated for 20 min under nitrogen. Further, $5 \mathrm{~mL}$ of $15 \%$ boron trifluoride, freshly prepared in methanol, was added to methylate the samples. After $2 \mathrm{~min}, 5 \mathrm{~mL}$ of heptane and $2 \mathrm{~mL}$ of saturated solution of sodium chloride were added and the sample was removed from the heating block (LTHS 250, Brnenska Druteva, Czech Republic). Then, $15 \mathrm{~mL}$ of heptane and $40 \mathrm{~mL}$ of saturated solution of sodium chloride were added to the extract of FAMEs, the mixture was shaken and phases were separated and washed subsequently with $40 \mathrm{~mL}$ of saturated solution of sodium chloride. Heptane phase was separated and anhydrous sodium sulfate was added. Prepared FAMEs were transferred into $50 \mathrm{~mL}$ volumetric flask together with $1 \mathrm{~mL}$ of methyl-undecanoate as the internal standard and replenished by hexan.

\subsection{GC Analysis of FAMEs}

Quantitative determinations of FAMEs were conducted according to [71] using a Shimadzu GC-2010 gas chromatograph (Shimadzu Corporation, Tokyo, Japan) with a flame ionization detector 
(FID) and capillary column HP-88 Agilent Technologies $(100 \mathrm{~m} \times 0.25 \mathrm{~mm})$ with a stationary phase (88\% cyanopropyl, aryl-polysiloxan) with the thickness of $0.2 \mu \mathrm{m}$. The injection volume was $1.0 \mu \mathrm{L}$, the temperature of injection port was $250{ }^{\circ} \mathrm{C}$ with the split ratio of $1: 100$ and nitrogen was used as a carrier gas, temperature program was $80{ }^{\circ} \mathrm{C} / 5 \mathrm{~min}, 200{ }^{\circ} \mathrm{C} / 30 \mathrm{~min}$, and $250{ }^{\circ} \mathrm{C} / 15 \mathrm{~min}$. Identification of FAMEs was performed by comparing their retention times with those of reference standards (mixture FAME Mix, SUPELCO, which included 37 FAMEs). For quantification of FAMEs, methyl-undecanoate (Sigma Aldrich Chemical Co., St. Louis, MO, USA) was used as the internal standard. The results of FAs were expressed as percentages of total FAMEs.

\subsection{Determination of Energy Contribution of Fatty Acid Groups of Vegetable Oils}

Amounts of fatty acid contents $(\mathrm{mg} / \mathrm{g})$ were converted to energy values using the conversion factor of $37.7 \mathrm{~kJ} / \mathrm{g}$ for fat and fatty acids, because of the evaluation of energy $\mathrm{E}(\mathrm{kJ} / \mathrm{g}$ oils $)$ derived from SFAs, MUFAs, PUFAs, n-3 and n-6 PUFAs of analyzed vegetable oils [61].

Further, energy contribution E (\% ERDI) of SFAs, PUFAs, n-3 PUFAs and n-6 PUFAs of analyzed oils to recommended dietary intakes for total fat $\left(\mathrm{E}_{\mathrm{RDI}}-37.7 \mathrm{~kJ} / \mathrm{g}\right)$ was calculated and it was expressed as an amount ( $\mathrm{g}$ ) of analyzed oils needed to cover reported recommended daily intakes for individual fatty acid groups. Values of energy contribution of fatty acid groups of analyzed oils were calculated from the maximal values of recommended daily intakes (ERDI) according to [15] for fatty acids (ERDI-SFAs - 10\% E; ERDI-PUFAs—11\% E; ERDI-n-3—2\% E and ERDI-n-6—9\% E).

\subsection{Statistical Evaluation of Impact of Fat Dietary Intake on the Number of CHD and CVD Mortality in Various Countries}

High number of CHD and CVD mortality in many Western countries is a serious problem caused by a complex of factors and the composition of dietary fat belongs to them. So, the evaluation of influence of dietary fats intakes on CHD and CVD mortality was also provided from the reported data of total fats, SFAs, MUFAs and PUFAs intakes among adults in different countries. Spearman's correlations between SFAs, MUFAs and PUFAs intakes and CHD and CVD mortality were computed. The significance level was set to 0.05 (5\%). Statistical analyses were conducted using QCExpert 3.3, TriloByte statistical software, Ltd. (Pardubice, Czech Republic).

\section{Conclusions}

A direct relationship between diet and human health was postulated by Hippocrates in the 4th BC. Energy balance is an important factor to maintain healthy body weight. Oversized intake of energy without adequate physical exertion leads to obesity and relating ailments that can result in serious diseases including CHD.

Vegetable oils are sources of important fatty acids, embracing all groups of SFAs, MUFAs, and PUFAs. However, their fatty acid composition varies according to the source plant or it depends on the technology process during their production. The study was focused on the oils obtained mostly by mechanical ways under the conditions that do not cause any changes in their chemical composition. From these results, it could be concluded that the majority of analyzed vegetable oils is characterized 
by higher amount of PUFAs, with especially higher amounts of n-6 PUFAs, so they could not be used as oils for daily consumption, but rather as oils to supplement specific PUFAs. The majority of them exceeded recommended intake of $11 \%$ ERDI. The highest contributions of PUFAs derived from $1 \mathrm{~g}$ oils were $786.8 \%$ ERDI and $626.3 \%$ ERDI in sunflower oil (SFL) and safflower oil (SAF), respectively. Thus, to cover recommended daily energy intake of PUFAs only $0.1 \mathrm{~g}$ of sunflower oil and $0.2 \mathrm{~g}$ of safflower oil, grape oil, silybum marianum oil or hemp oil is needed. These oil amounts were calculated in relation to the maximal values of recommended daily energy intakes for PUFAs.

Statistical analyses using Spearman's correlation has not confirmed any direct significant relationship between CHD and CVD mortalities and intakes of total fats, SFAs, MUFAs and PUFAs in various countries. Studies focused on the relationship between total fats, SFAs and PUFAs intakes and CHD have to be multifactorial because the presence of many environmental factors and personal conditions that have simultaneous impacts on the human metabolic pathways.

\section{Acknowledgments}

This study was funded by internal grant agency of Tomas Bata University in Zlín, project no. IGA/FT/2015/010. We would also like to thank Ms. Lucie Slovackova for providing some analyses of FAs contents.

\section{Author Contributions}

All authors designed research; Jana Orsavova, Ladislava Misurcova, and Jarmila Vavra Ambrozova performed the research, analyzed the data and wrote the paper. Jana Orsavova, Ladislava Misurcova, Jarmila Vavra Ambrozova, Robert Vicha and Jiri Mlcek read and approved the final manuscript.

\section{Conflicts of Interest}

The authors declare no conflict of interest.

\section{References}

1. Mišurcová, L.; Vávra Ambrožová, J.; Samek, D. Seaweed lipids as nutraceuticals. Adv. Food Nutr. Res. 2011, 64, 339-355.

2. Brenna, J.T. Efficiency of conversion of $\alpha$-linolenic acid to long chain n-3 fatty acids in man. Curr. Opin. Clin. Nutr. 2002, 5, 127-132.

3. Burdge G.C.; Calder, P.C. Conversion of $\alpha$-linolenic acid to longer-chain polyunsaturated fatty acids in human adults. Reprod. Nutr. Dev. 2005, 45, 581-597.

4. Burdge, G.C.; Wootton, S.A. Conversion of eicoapentaenoic, docosapentaenoic and docosahexaenoic acids in young women. Br. J. Nutr. 2002, 88, 411-420.

5. Brenna, J.T.; Salem, J.N.; Sinclair, A.J.; Cunnane, S.C. $\alpha$-Linolenic acid supplementation and conversion to n-3 long-chain polyunsaturated fatty acids in humans. Prostaglandins Leukot. Essent. Fat. Acids 2009, 80, 85-91.

6. Huang, Ch.; Freter, C. Lipid metabolism, apoptosis and cancer therapy. Int. J. Mol. Sci. 2015, 16, 924-949. 
7. Gatek, J.; Vrana, D.; Melichar, B.; Vazan, P.; Kotocova, K.; Kotoc, J.; Dudesek, B.; Hnatek, L.; Duben, J. Significance of the resection margin and risk factors for close or positive resection margin in patients undergoing breast-conserving surgery. J. BUON 2012, 17, 452-456.

8. Simmons, G.E.; Pruitt, W.M.; Pruitt, K. Diverse roles of SIRT1 in cancer biology and lipid metabolism. Int. J. Mol. Sci. 2015, 16, 950-965.

9. Gatek, J.; Vrana, D.; Hnatek, L.; Bakala, J.; Dudesek, B.; Duben, J.; Musil, T. Sentinel node biopsy and neoadjuvant chemotherapy in the treatment of breast cancer. J. BUON 2012, 17, 265-270.

10. Proust, F.; Lucas, M.; Deawailly, É. Fatty acid profiles among the Inuit of Nunavi: Current status and temporal change. Prostaglandins Leukot. Essent. Fat. Acids 2014, 90, 159-167.

11. Bozza, P.T.; Viola, J.P.B. Lipid droplets in inflammation and cancer. Prostaglandins Leukot. Essent. Fat. Acids 2014, 90, 159-167.

12. Gard, M.L.; Thomson, A.B.R.; Clandinin, M.T. Effect of dietary cholesterol and/or $\omega 3$ fatty acids on lipid composition and $\Delta 5$-desaturase activity of rat liver microsomes. J. Nutr. 1988, 118 , 661-668.

13. Brenner, R.R.; Bernasconi, A.M.; González, M.S.; Rimoldi, O.J. Dietary cholesterol modulates $\Delta 6$ and $\Delta 9$ desaturase mRNAs and enzymatic activity in rats fed a low-EFA diet. Lipids 2002, 37, $375-383$.

14. Landau, J.M.; Sekowski, A.; Hamm, M.W. Dietary cholesterol and the activity of stearoyl CoA desaturase in rats: Evidence for an indirect regulatory effect. Biochim. Biophys. Acta 1997, 3, 349-357.

15. FAO/WHO. Fats and Fatty Acids in Human Nutrition. Report of an Expert Consultation; FAO/WHO: Geneva, Switzerland, 2010.

16. Tvrzická, E.; Staňková, B.; Vecka, M.; Žák, A. Fatty acids 1. Occurrence and biological significance (In Czech). Cas. Lek. Cesk. 2009, 148, 16-24.

17. Flachs, P.; Horakova, O.; Brauner, P.; Rossmeisl, M.; Pecina, P.; Franssen-van Hal, N.; Ruzickova, J.; Sponarova, J.; Drahota, Z.; Vlcek, C.; et al. Polyunsaturated fatty acids of marine origin upregulate mitochondrial biogenesis and induce $\beta$-oxidation in white fat. Diabetologia 2005, 48, 2365-2375.

18. Kinsella, J.E.; Lokesh, B.; Stone, R.A. Dietary n-3 polyunsaturated fatty acids and amelioration of cardiovascular disease: Possible mechanisms. Am. J. Clin. Nutr. 1990, 52, 1-28.

19. Weiss, L.A.; Barrett-Connor, E.; von Mühlen, D. Ratio of n-6 to n-3 fatty acids and bone mineral density in older adults: The Rancho Bernardo Study. Am. J. Clin. Nutr. 2005, 81, 934-938.

20. Hu, F.B.; Manson, J.A.E.; Willett, W.C. Types of dietary fat and risk of coronary heart disease: A critical review. J. Am. Coll. Nutr. 2001, 20, 5-19.

21. Mobraten, K.; Haug, T.M.; Kleiveland, C.R.; Lea, T. Omega-3 and omega-6 PUFAs induce the same GPR120-mediated signaling events, but with different kinetics and intensity in Caco-2 cells. Lipids Health Dis. 2013, 12, 101-107.

22. Salem, J.; Vandal, M.N.; Calon, F. The benefit of docosahexaenoic acid for the adult brain in aging and dementia. Prostaglandins Leukot. Essent. Fat. Acids 2015, 92, 15-22.

23. Ramsden, C.; Mann, J.D.; Faurot, K.R.; Lynch, C.; Imam, S.T.; MacIntosh, B.A.; Hibbeln, J.R.; Loewke, J.; Smith, S.; Coble, R.; et al. Low omega-6 vs. low omega-6 plus high omega-3 dietary intervention for Chronic Daily headache: Protocol for a randomized clinical trial. Trials 2011, 12, $1-11$. 
24. Puri, B.K.; Martins, J.G. Which polyunsaturated fatty acids are active in children with attention-deficit hyperactivity disorder receiving PUFA supplementation? A fatty acid validated meta-regression analysis of randomized controlled trials. Prostaglandins Leukot. Essent. Fat. Acids 2014, 90, 179-189.

25. Simopoulos, A.P. Omega-3 fatty acids in inflammation and autoimmune diseases. J. Am. Coll. Nutr. 2002, 21, 495-505.

26. Kris-Etherton, P.M.; Harris, W.S.; Appel, L.J. Fish consumption, fish oil, omega-3 fatty acids, and cardiovascular disease. Circulation 2002, 106, 2747-2757.

27. Kris-Etherton P.M.; Grieger, J.A.; Etherton, T.D. Dietary reference intakes for DHA and EPA. Prostaglandins Leukot. Essent. Fat. Acids 2009, 81, 99-104.

28. Maehre, H.K.; Malde, M.K.; Eilertsen, K.E.; Elvevoll, E.O. Characterization of protein, lipid and mineral contents in common Norwegian seaweeds and evaluation of their potential as food and feed. J. Sci. Food Agric. 2014, 94, 3281-3290.

29. Hertting, G.; Seregi, A. Formation and function of eicosanoids in the central nervous system. Ann. N. Y. Acad. Sci. 1989, 559, 84-89.

30. Pokorný, J.; Dubská, L. Technology of Lipids (In Czech); Publisher of Technical Literature: Praque, Czech Republic, 1986; pp. 1-452.

31. Zambiazi, R.C.; Przybylski, R.; Zambiazi, M.W.; Mendonca, C.B. Fatty acid composition of vegetable oils and fats. B. CEPPA Curitiba 2007, 25, 111-120.

32. Kostik, V.; Memeti, S.; Bauer, B. Fatty acid composition of edible oils and fats. J. Hyg. Eng. Des. 2013, 4, 112-116.

33. Rajah, K.K. Fats in Food Technology; Sheffield Academic Press: Sheffield, UK, 2002; pp. 1-379.

34. Alfawaz, M.A. Chemical composition and oil characteristics of pumpkin (Cucurbita maxima) seed kernels. Food Sci. Agric. Res. Center 2004, 129, 5-18.

35. Fathi-Achachlouei, B.; Azadmard-Damirchi, S. Milk thistle seed oil constituents from different varieties grown in Iran. J. Am. Oil Chem. Soc. 2009, 86, 643-649.

36. El-Mallah, M.H.; El-Shami, S.M.; Hassanein, M.M. Detailed studies on some lipids of Silybum marianum(L.) seed oil. Grasas Aceites 2003, 54, 397-402.

37. Kamal-Eldin, A.; Anderson, R. A multivariate study of the correlation between tocopherol content and fatty acid composition in vegetable oils. J. Am. Oil Chem. Soc. 1997, 74, 375-380.

38. Denke, M.A.; Grundy, S.M. Comparison of effects of lauric acid and palmitic acid on plasma lipids and lipoproteins. Am. J. Clin. Nutr. 1992, 56, 895-898.

39. Zock, P.L.; de Vries, J.H.M.; Katan, M.B. Impact of myristic acid versus palmitic acid on serum lipid and lipoprotein levels in healthy women and men. Arterioscler. Thromb. Vasc. 1994, 14, 567-575.

40. Mensink, R.P.; Zock, P.L.; Kester, A.D.M.; Katan, M.B. Effects of dietary fatty acids and carbohydrates on the ratio of serum total to HDL cholesterol and on serum lipids and apolipoproteins: A meta-analysis of 60 controlled trials ${ }^{1-3}$. Am. J. Clin. Nutr. 2003, 77, 1146-1155.

41. Lawrence, G.D. Dietary fats and health: Dietary recommendations in the context of scientific evidence. Adv. Nutr. 2013, 4, 294-302.

42. Pala, V.; Krogh, V.; Muti, P.; Chajés, V.; Riboli, E.; Micheli, A.; Saadatian, M.; Sieri, S.; Berrino, F. Erythrocyte membrane fatty acids and subsaquent breast cancer: A prospective Italian study. J. Natl. Cancer Inst. 2001, 93, 1088-1095. 
43. Kim, H.; Youn, K.; Yun, E.Y.; Hwang, J.S.; Jeong, W.S.; Ho, Ch.T.; Jun, M. Oleic acid ameliorates $\mathrm{A} \beta$-induced inflammation by downregulation of COX-2 and iNOS via NFkB signaling pathway. J. Funct. Foods 2015, 14, 1-11.

44. Yongmanitchai, W.; Ward, O.P. Positional distribution of fatty acids, and molecular species of polar lipids, in the diatom Phaeodactylum tricornutum. J. Gen. Microbiol. 1993, 139, 465-472.

45. Vávra Ambrožová, J.; Mišurcová, L.; Vícha, R.; Machů, L.; Samek, D.; Baroň, M.; Mlček, J. Sochor, J.; Juř́ková, T. Influence of extractive solvents on lipid and fatty acids content of edible freshwater algal and seaweed products and green microalga Chlorella kessleri and cyanobacteria Spirulina platensis. Molecules 2014, 19, 2344-2360.

46. Mišurcová, L. Chemical composition of seaweeds. In Handbook of Marine Macroalgae: Biotechnology and Applied Phycology; Kim, S.K., Ed.; John Wiley \& Sons Ltd.: Chichester, UK, 2011; pp.173-192.

47. De Caterina, R.; Basta, G. n-3 Fatty acids and the inflammatory response-Biological background. Eur. Heart J. Suppl. 2001, 3, D42-D49.

48. AI, F.F.; Bin, J.; Zhang, Z.M.; Huang, J.H.; Wang, J.B.; Liang, Y.Z.; Yu, L.; Yang, Z.Y. Application of random forests to select quality vegetable oils by their fatty acid composition. Food Chem. 2014, 143, 473-478.

49. Abedi, E.; Sahari, M.A. Long-chain polyunsaturated fatty acid sources and evaluation of their nutritional and functional properties. Food Sci. Nutr. 2014, 2, 443-463.

50. De Caterina, R.; Liao, J.K.; Libby, P. Fatty acid modulation of endothelial activation. Am. J. Clin. Nutr. 2000, 71, 213S-223S.

51. Narayan, B.; Miyashita, K.; Hosakawa, M. Physiological effects of eicosapentaenoic acid (EPA) and docosahexaenoic acid (DHA) —A review. Food Rev. Int. 2006, 22, 291-307.

52. Sugano, M.; Hirahara, F. Polyunsaturated fatty acids in the food chain in Japan. Am. J. Clin. Nutr. 2000, 71, 189S-196S.

53. Cordain, L.; Eaton, S.B.; Miller, J.B.; Mann, N.; Hill, K. The paradoxical nature of hunter-gatherer diets: Meat-based, yet non-atherogenic. Eur. J. Clin. Nutr. 2002, 56, 542-552.

54. Sundram, K.; Ismail, A.; Hayes, K.C.; Jeyamalar, R.; Pathmanathan, R. Trans (elaidic) fatty acids adversely affect the lipoprotein profile relative to specific saturated fatty acids in humans ${ }^{1,2}$. J. Nutr. 1997, 127, 514S-520S.

55. Elmadfa, I.; Kornsteiner, M. Dietary fat intake-A global perspective. Ann. Nutr. Metab. 2009, $54,8-14$.

56. Allender, S.; Scarborough, P.; Peto, V.; Rayner, M.; Leal, J.; Luengo-Fernandez, R.; Gray, A. European Cardiovascular Disease Statistics; European Heart Network: Brussels, Belgium, 2008.

57. Hay, D.R. Cardiovascular disease in New Zealand, 2004: A summary of recent statistical information. In Technical Report: To Medical and Allied Professions; Heart Foundation: Auckland, New Zealand, 2004.

58. Norman, R.; Bradshaw, D.; Schneider, M.; Pieterse, D.; Groenewald, P. What are the top causes of death in South Africa? In Proceedings of the South African Medical Research Council, Cape Town, South Africa, 2006.

59. Iso, H. Changes in coronary heart disease risk among Japanese. Circulation 2008, 118, 2725-2729. 
60. Roger, V.L.; Go, A.S.; Lloyd-Jones, D.M.; Benjamin, E.J.; Berry, J.D.; Borden, W.B.; Bravata, D.M.; Dai, S.; Ford, E.S.; Fox, C.S.; et al. Heart disease and stroke statistics-2012 update: A report from the American Heart Association. Circulation 2011, 125, 1-221.

61. Harika, R.K.; Eilander, A.; Osendarp, S.J.M.; Zock, P.L. Intake of fatty acids in general Populations Worldwide Does Not Meet dietary recommendations to prevent Coronary Heart Disease: A systematic review of data from 40 countries. Ann. Nutr. Metab. 2013, 63, 229-238.

62. Oh, K.; Hu, F.B.; Manson, J.E.; Stampfer, M.J.; Willett, W.C. Dietary fat intake and risk of coronary heart disease in women: 20 Years of follow-up of the nurses'health study. Am. J. Epidemiol. 2005, $161,672-679$.

63. Siri-Tarino, P.W.; Sun, Q.; Hu, F.B.; Krauss, R.M. Meta-analysis of prospective cohort studies evaluating the association of saturated fat with cardiovascular disease ${ }^{1-5}$. Am. J. Clin. Nutr. 2010, 91, 535-546.

64. Chowdhury, R.; Warnakula, S.; Kunutsor, S.; Crowe, F.; Ward, H.A.; Johnson, L.; Franco, O.H.; Butterworth, A.S.; Forouhi, N.G.; Thompson, S.G.; et al. Association of dietary, circulating, and supplement fatty acids with coronary risk. Ann. Intern. Med. 2014, 160, 398-406.

65. Czernichow, S.; Thomas, D.; Bruckert, E. n-6 Fatty acids and cardiovascular health: A review of the evidence for dietary intake recommendations. Br. J. Nutr. 2010, 104, 788-796.

66. Micha, R.; Khatibzadeh, S.; Shi, P.; Fahimi, S.; Lim, S.; Andrews, K.G.; Engell, R.E.; Powles, J.; Ezzati, M.; Mozaffarian, D. Global, regional, and national consumption levels of dietary fats and oils in 1990 ad 2010: A systematic analysis including 266 country-specific nutrition surveys. Br. Med. J. 2014, 348, 1-20.

67. Jakobsen, M.U.; O’Reilly, E.J.; Heitmann, B.L.; Pereira, M.A.; Balter, K.; Fraser, G.E.; Goldbourt, U.; Hallmans, G.; Knekt, P.; Liu, S.; et al. Major types of dietary fat and risk of coronary heart diseases: A pooled analysis of 11 cohort studies $^{1-3}$. Am. J. Clin. Nutr. 2009, 89, 1425-1432.

68. Abbasi, F.; Brown, B.W.; Lamendola, C.; McLaughlin, T.; Reaven, G.M. Relationship between Obesity, Insulin Resistance, and Coronary Heart Disease Risk. J. Am. Coll. Cardiol. 2002, 40, 937-943.

69. ČSN CEN ISO/TS 17764-1. Animal Feeding Stuffs-Determination of the Content of Fatty Acids-Part 1: Preparation of Methyl Esters. Czech Standards Institute: Prague, Czech Republic, 2007; pp. 1-20.

70. Macedo, L.F.A.; Lacerda, E.C.Q.; Silva, R.R.; Simionato, J.I.; Pedrao, M.R.; Coro, F.A.G.; de Souza, N.E. Implication of method chosen for analysis of fatty acids in meat: A review. Am. J. Agric. Biol. Sci. 2012, 7, 278-284.

71. ČSN CEN ISO/TS 17764-2. Animal Feeding Stuffs-Determination of the Content of Fatty Acids-Part 2: Gas Chromatographic Method; Czech Standards Institute: Prague, Czech Republic, 2007; pp. 1-24.

(C) 2015 by the authors; licensee MDPI, Basel, Switzerland. This article is an open access article distributed under the terms and conditions of the Creative Commons Attribution license (http://creativecommons.org/licenses/by/4.0/). 